\title{
Relationship between tinnitus and sexual dysfunction
}

\author{
Gül Soylu Özler ${ }^{a^{*}}$, Serkan Özler ${ }^{b}$ \\ ${ }^{a}$ Department of Otorhinolaryngology, Faculty of Medicine, Mustafa Kemal University, Hatay, Turkey \\ ${ }^{b}$ Urology Clinic, Antakya State Hospital, Hatay, Turkey
}

\section{ARTICLE INFO}

\section{Article History}

$\begin{array}{ll}\text { Received } & 20 / 02 / 2014 \\ \text { Accepted } & 05 / 05 / 2014\end{array}$

\section{* Correspondence to: \\ Gül Soylu Özler \\ Department of Otorhinolaryngology, \\ Faculty of Medicine, \\ Mustafa Kemal University, Hatay, Turkey \\ e-mail: soylugul@yahoo.com}

\section{Keywords:}

International Index of Erectile function

Questionnaire

Quality of sexual life

Quality of life

Tinnitus

\section{ABSTRACT}

The aim of this study is to evaluate the quality of sexual life of males with and without tinnitus. Fourty patients with a complaint of tinnitus and 40 control subjects with no evidence of ear pathology were included. International Index of Erectile Function questionnaire (IIEF) was used to evaluate the quality of sexual life of the subjects. The mean scores of erectile function, orgasmic function, sexual desire, intercourse satisfaction and overall satisfaction for tinnitus group were significantly lower than that for control group $(\mathrm{p}=0.0001)$. There was no correlation between tinnitus duration and IIEF scores $(p \leq 0.05)$. There was no correlation between tinnitus severity and IIEF scores $(p \leq 0.05)$. Tinnitus is not only an otologic problem but also has negative effects on quality of life. The clinician must consider these concomitting social, sexual and sleep disturbances and help the patient to cope with these problems.

J. Exp. Clin. Med., 2014; 31:67-69

\section{Introduction}

Tinnitus is the perception of sound in the absence of any external acoustic stimuli. Tinnitus is a common condition with prevalence rates between $2.4 \%$ and $20.1 \%$ (Moller et al., 2010). The extent to which tinnitus impairs quality of life is highly variable. Most of the studies declared that tinnitus strongly effects psychological and social profile of the patients and decreases their quality of life (Meikle et al., 1984; Bauch et al., 2003; Crönlein et al., 2007). Some people are severely impaired and may even become suicidal, whereas some remain unaffected by the phantom sounds (Turner et al., 2007). Therefore, tinnitus is not only a medical problem but also must be considered as a social impediment. Although several studies have investigated the psychosocial effects of tinnitus, the effects of tinnitus on quality of sexual life has been neglected.

In this study, we used the International Index of Erectile Function questionnaire (IIEF) to evaluate the quality of sexual life of males with tinnitus and compared the results with an age and sex matched group of healthy men.

\section{Materials and methods Study population}

Forty patients with a complaint of tinnitus for at least 6 months and 40 control subjects with no evidence of ear pathology completed the study. Males between 20-50 years old; with a complaint of tinnitus; married and able to complete the study were included. The exclusion criterias were: subjects with an ear pathology except for hearing loss and hearing aid; ages below 20 years or over 50 years; single or widowed; lack of mental capacity; previous any other disease; previous sexual or psychiatric disorders; usage of any medication; Body Mass Index (BMI) of $30 \mathrm{~kg} / \mathrm{m}^{2}$ or more; a history of ear or urologic surgery, alcohol dependence and smoking. The control group were healthy, adult, married males who have normal otorhinolaryngologic examination. Age, gender, duration of tinnitus were listed on a chart. Tinnitus severity was measured by Tinnitus Handicap Inventory (THI) (for tinnitus group). IIEF was used to evaluate the quality of sexual life of the subjects. 


\section{Questionnaires}

Quality of sexual life was assessed using the IIEF. This questionnaire has been widely used to evaluate male sexual function. It consists of 15 items grouped into 5 sexual function domains: erectile function (six questions); orgasmic function (two questions); sexual desire (two questions); sexual intercourse satisfaction (three questions); and overall satisfaction (two questions) (Jakes et al.,1985). Each question was scored from 1 to 5 . The individual scores in each domain were rated to indicate the degree of clinical dysfunction; rating numbers were defined as follows: $5=$ no dysfunction, 4=mild dysfunction, 3=mild-to-moderate, $2=$ moderate and $1=$ severe. Lower question scores indicates higher degrees of dysfunction, while higher scores means healthier sexual function.

\section{Tinnitus Handicap Inventory (THI)}

The purpose of this scale is to identify the problems that tinnitus causing the patient. Total THI score is the sum of the responses of 25 questions. Number of 'Yes' responses x $4+$ number of 'Sometimes' responses $\mathrm{x} 2$ gives the total THI score. The higher score shows more handicap.

\section{Procedures}

This study is carried out by the collabration of otorhinolaryngolgy and urology departments between June 2013 and February 2014. Questionnaires were administered to all participants within a few days. Ethics committee approval was obtained and the study was conducted adhering to the Declaration of Helsinki. Informed consent was obtained from all participants

\section{Statistical Analysis}

Statistical analysis was performed using the Statistical Package for the Social Sciences (SPSS) 13.0 evaluation for Windows. Normal distribution of continued variables were tested with Kolmogorov-Smirnov test. Chi-square test was used for comparisons between categorical variables. Kruskal-Wallis test and Mann-Whitney U tests were used for continued variables when comparing the groups. The correlations between continued variables were assessed by Pearson correlation coefficient. The statistically significant level was accepted as a $\mathrm{p}$ value $<0.05$.

\section{Results}

\section{Demographic data}

Fourty tinnitus patients and 40 control subjects without tinnitus completed the study. Mean age of the tinnitus group was $33.95 \pm 6.98$, whereas it was $33.28 \pm 6.30$ for control group. The groups were similar in terms of age $(\mathrm{p}=0.651)$.

\section{Tinnitus and quality of sexual life}

The mean scores of erectile function, orgasmic function, sexual desire, intercourse satisfaction and overall satisfaction are shown in Table 1. The mean scores for tinnitus group were significantly lower than those control group $(\mathrm{p}=0.0001)$.

\section{Tinnitus duration and quality of sexual life}

The mean tinnitus duration in tinnitus group were 8.30 \pm 3.42 years. There was no correlation betwen tinnitus duration and IIEF scores $(\mathrm{p} \leq 0.05)$.
Table 1. Scores for international index of erectile function questionnaire between tinnitus group and control group

\begin{tabular}{lccc} 
& Tinnitus group & $\begin{array}{c}\text { Control } \\
\text { group }\end{array}$ & $\boldsymbol{p}$-value \\
\hline Erectile function & $21.85 \pm 3.10$ & $25.53 \pm 3.33$ & 0.0001 \\
Orgasmic function & $8.13 \pm 1.30$ & $9.32 \pm 0.76$ & 0.0001 \\
Sexual desire & $6.20 \pm 2.07$ & $8.85 \pm 1.51$ & 0.0001 \\
Intercourse satisfaction & $10.83 \pm 1.70$ & $12.65 \pm 1.68$ & 0.0001 \\
Overall satisfaction & $6.93 \pm 1.55$ & $8.93 \pm 1.16$ & 0.0001 \\
\hline
\end{tabular}

Tinnitus Handicap Inventory and quality of sexual life There was no correlation betwen THI scores and IIEF scores $(\mathrm{p} \leq 0.05)$.

\section{Discussion}

Tinnitus is a very common condition among general population that has negative effects on quality of life and psychosocial profile of a person. A study showed that $43 \%$ of patients with tinnitus had impaired quality of life or a high level of distress or both (Bauch et al., 2003). Sleep disturbances in tinnitus patients have been reported by several studies previously. The prevelance of sleep disturbance among tinnitus patients have been reported to be between $25 \%-60 \%$ and found to be one of the three major complaints (Jakes et al., 1985; Axelsson and Ringdahl, 1989; Sanchez and Stephens, 2000). Furthermore, many tinnitus patients are known to suffer from insomnia (Crönlein et al., 2007), which has a considerable impact on the quality of life (Meikle et al., 1984).

Sexual life is mostly kept secret, it is thought to be shameful to talk about it. However, sexual life is one of the most important factors effecting the quality of life. For this reason, studies evaluating quality of sexual life must not be neglected.

In this study, the scores of erectile function, orgasmic function, sexual desire, intercourse satisfaction and overall satisfaction for tinnitus group were significantly lower than that of control group, that means tinnitus effects the quality of sexual life of individuals negatively. Moreover, the relationship between tinnitus severity and tinnitus duration were evaluated. The results declared no correlation between tinnitus duration and IIEF scores. Also there was no correlation between tinnitus severity and IIEF scores. In the literature, there is only one study about sexual life of tinnitus sufferers. They reported that sexual disturbance was seen in tinnitus sufferers in the early period of the disease. Over time, they become used to living with their tinnitus, and no loss in sexual performance was seen (Muluk et al., 2007).

The effects of vertigo, hearing loss, halitosis and obstructive sleep apnea (OSAS) on sexual health have been previously investigated. In patients with Ménière's disease, erectile dysfunction has been reported in men (Zapata and Lopez-Escames, 2011). Similarly, in patients with hearing loss, they found twice the prevalence of erectile dysfunction compared with control subjects (Bakır et al., 2012). In another study with hearing loss patients, the orgasmic function, sexual desire and overall satisfaction domains were negatively affected whereas the scores for erectile function and intercourse satisfaction were not affected (Ozler and Ozler, 2013). Also, males with halitosis reported lower scores for all function domains that means poorer sexual life (Ozler and Ozler, 2014). Moreover, it has been reported that OSAS 
declined quality of sexual life and a significant improvement was detected with apnea treatment (Ceylan et al., 2013).

Tinnitus is not only an otologic problem but also has negative effects on quality of life. The otorhinolaryngologist must consider these concomitting social, sexual and sleep disturbances and help the patient to cope with these problems.

\section{REFERENCES}

Axelsson, A., Ringdahl, A., 1989. Tinnitus-a study of its prevalence and characteristics. Br J. Audiol. 23, 53-62.

Bakır, S., Penbegül, N., Gün, R., 2012. Relationship between hearing loss and sexual dysfunction. J. Laryngol. Otol. 20, 1-6. doi: 10.1017/ S0022215112002952.

Bauch, C.D., Lynn, S.G., Williams, D.E., Mellon, M.W., Weaver, A.L., 2003. Tinnitus impact: Three different measurement tools. J. Am. Acad. Audiol. 14, 181-187.

Ceylan, C., Odabaş, O., Yığman, M., Doğan, S., Yüksel, S., 2013. Does the treatment of sleep apnea improve the sexual performance in men with obstructive sleep apnea syndrome? J. Clin. Anal. Med. 4, 9-12.

Crönlein, T., Langguth, B., Geisler, P., Hajak, G., 2007. Tinnitus and insomnia. Prog. Brain. Res. 166, $227-233$.

Jakes, S.C., Hallam, R.S., Chambers, C., 1985. A factor analytical study of tinnitus complaint behaviour. Audiology. 24, $195-206$.

Meikle, M.B., Vernon, J., Johnson, R.M., 1984. The perceived severity of tinnitus. Some observations concerning a large population of tinnitus clinic patients. Otolaryngol Neck Surg. 92, 689-696.

Moller, A.R., Langguth, B., DeRidder, D., Kleinjung, T., 2010. Textbook of tinnitus: Diagnosis and treatment. 1st edition. New York: Springer. p.p.786.

Muluk, N.B., Basar, M.M., Oguzturk, O., Dikici, O., 2007. Does subjective tinnitus cause sexual disturbance? J. Otolaryngol. $36,77-82$.

Ozler, G.S., Ozler, S., 2013. Quality of social and sexual life in males with hearing loss. Int. Adv. Otol. 9, 211-218.

Ozler, G.S., Ozler, S., 2014. Does halitosis effect the sexual life of males with halitosis? J. Clin. Anal. Med. 1-3 doi:10.4328/JCAM.2230.

Sanchez, L., Stephens, D., 2000. Survey of the perceived benefits and shortcomings of a specialisttinnitus clinic. Audiology. 39, $333-339$.

Turner, O., Windfuhr, K., Kapur, N., 2007. Suicide in deaf populations: A literatüre review. Ann. Gen. Psychiatry. 6, 26.

Zapata, C., López-Escámez, J.A., 2011. A pilot study of sexual health in patients with Ménière's disease. Acta Otorrinolaringol Esp. 62, 119-125. doi: 10.1016/j.otorri.2010.09.010. 